International Journal of Engineering \& Technology, $7(2.8)(2018) 42-46$
International Journal of Engineering \& Technology
Website: www.sciencepubco.com/index.php/IJET
Research Paper

\title{
An affine view and illumination invariant iterative image matching approach for face recognition
}

\author{
D. Rajasekhar ${ }^{1 *}$, T. Jayachandra Prasad ${ }^{2}$, K. Soundararajan ${ }^{3}$ \\ ${ }^{1}$ Research Scholar, Department of ECE, JNTUA, Ananthapuramu, Andhra Pradesh-515002 \\ ${ }^{2}$ Professor and Principal, RGMCET, Nandyal, Andhra Pradesh, India-518501 \\ 3 Professor and Dean of R\&D, TKREC, Hyderabad, Telangana-500097 \\ *Corresponding author E-mail: dkethanaraj@gmail.com
}

\begin{abstract}
Feature detection and image matching constitutes two primary tasks in photogrammetric and have multiple applications in a number of fields. One such application is face recognition. The critical nature of this application demands that image matching algorithm used in recognition of features in facial recognition to be robust and fast. The proposed method uses affine transforms to recognize the descriptors and classified by means of Bayes theorem. This paper demonstrates the suitability of the proposed image matching algorithm for use in face recognition appli-cations. Yale facial data set is used in the validation and the results are compared with SIFT (Scale Invariant Feature Transform) based face recognition approach.
\end{abstract}

Keywords: Face Recognition; Iterative Approach; Bayes; Yale; SIFT.

\section{Introduction}

One computer application that has garnered much attention recently is the automatic face recognition from digital still images, due to its multi-purpose nature. [1], [2].Here, using an face image of some known people and their database, the identity of the face is determined. Although, there is an availability of wide range of literature, including some recent works with good output on difficult datasets, face recognition still remains an unsolved problem [3].The face recognition methods are categorized as holistic based and feature based methods. The method that uses global information from the images to recognize face identity is classified as holistic face recognition methods. The global information is nothing but a set of features, derived directly from the pixels of the images that capture variance among distinct faces. The methods such as the Eigen faces method [4] and the Fisher's Linear Discriminate (FLD) [5] are holistic based face recognition methods. An alternative approach to this method was proposed, known as the local feature based face recognition method. This method is now an active area of research in this field. Out of the various local features used, the local binary patterns, histogram of oriented gradients and Gabor wavelets [6] are some of the prominent ones. The Lowe's work on an object recognition using SIFT (Scale Invariant Feature Transform) descriptors[7] have been used by multiple authors in fields like robot navigation [8], scene classification [9], and also face recognition [10], [11], [12], [13], [14 ].

A general approach to all these methods is quite same; first, a number of key points from the images are extracted, then each key point is computed using a local descriptor, finally, each point descriptor in the test image is matched with all the descriptors extracted from the image database, to recognize the identity of the test image. The class to which the input image is to be assigned depends on the output of the matching procedure. One of the most important problem that need special attention while using local features for face recognition is the possibility of false matched key-points. This problem is tackled by most of the local feature approaches; by adopting a grid based matching strategy for face recognition. It works by establishing sub-regions on the face images, so that only the descriptors in between these sub- regions are compared for matching. By this way the number of wrong matches can be reduced(without elimination). However, the images need to be preregistered and it makes the application of this method a bit difficult for datasets with random views [15]. The variable illumination still significantly affects the identification of key points, as the key point detector intrinsic to the SIFT techniques may change to with the illumination [13].

In this approach an iterative based image matching approach is used in the application of face recognition. At the beginning it finds the affine invariant data points in all mages. Then descriptors are assigned and the calculation of maximum disparity range is followed. Next the area around all key points is selected Bayes theory is applied for assigning the initial probabilities. Then match the key points in two images. This method is applied on facial images from Yale's facial database. The results are compared with a face recognition approach employing SIFT.

\section{Feature detection in scale-space}

SIFT key points play an important role in computer vision due to their stability to scale changes, dependency to the illuminations and rotations of images [7]. These features make SIFT key points strong enough against the affine distortions and change of poses. The SIFT [16] process is carried out in 2 steps; first step includes detection of key points in scale-space pyramid and then key point description. Since the SIFT features are less expensive features [17] and [18] have been proposed. 


\section{Yale face data base}

The Yale face data face [19] comprises 165 grayscale images in GIF format of 15 individuals. It has 11 images per subject having different facial expression or configuration which includes centerlight, w/glasses, happy, left-light, w/no glasses, normal, right-light, sad, and sleepy, surprised, and wink. In this work 10 images per subject are considered for testing and analysis. Different images from a single subject used in the work are illustrated in the Fig.1.
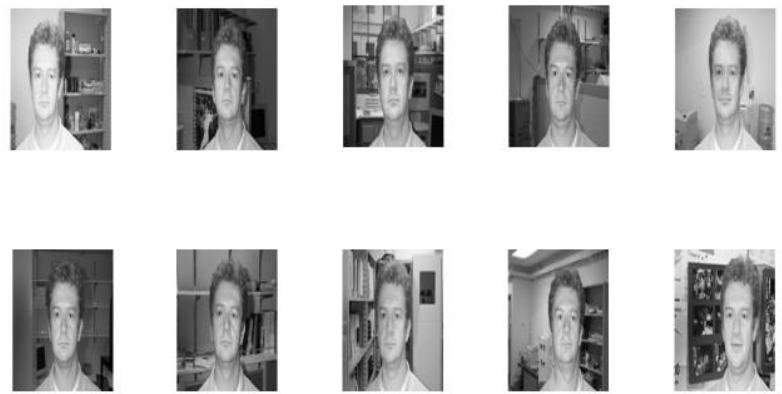

Fig. 1: Sample Images from the Yale Face Data Set.

In the test images, the feature detection algorithms extract features from the back ground and other objects that are not related to face. These background features can result in false matches. In order to avoid this, the face part of the image is cropped using Viola Jones Object Detection algorithm [20]. The object detection trained using OpenCV trained classifiers [21]. This algorithm locates the face region and then crops that particular region. This results in the reduction of unwanted features and also the number of features / descriptors. This eventually increases the matching speed as well the accuracy of matching. Features identified for cropped and uncropped images are illustrated using the Fig.2 and Fig.3
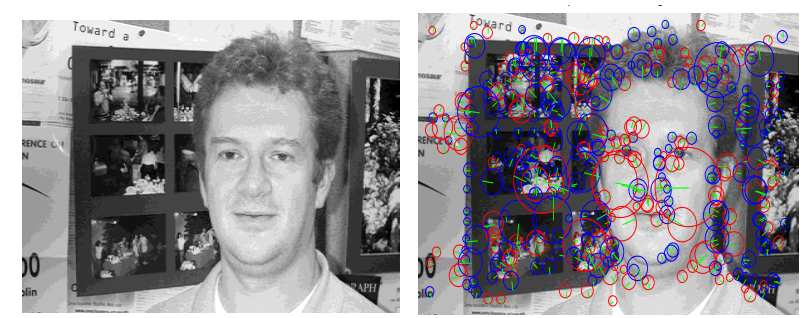

Fig. 2: Uncropped Image and Descriptors Identified for the Image.

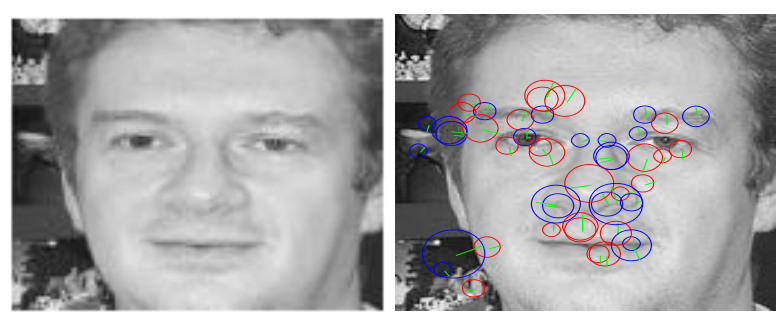

Fig. 3 : Cropped Image and Decriptors Identfied.

\section{Proposed method}

The steps of the proposed method are as follows.

Step 1: Select key points using ASIFT in both images.

Step 2: Compute the descriptor for every key point.

Step 3: Select an area around every right key point node.

Step 4: Find all the key points in the area selected in step 3.

Step 5: The above two steps are accomplished for all the key points in both images.

Step 6: Pair up the left key point node $b_{i}$ with every right key point node $d_{i}$.

Step 7: Calculate the Euclidean distance between key point pair.

Step 8: Calculate the weight to all key points $d_{i}$ as

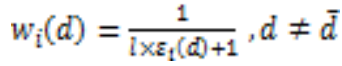

1 is a positive constant. $W_{i}(d)$ will be in the the range of 0 to 1 and weight is inversely proportional to Euclidean distance.

Step 9: For every category set $d, \bar{d}$ is undefined disparity category. Step 10: Initial probability for undefined category is given as

$p_{i}^{0}(d)=1-\max _{d \neq d}\left(w_{i}(d)\right)$

Step 11: Apply Bayes rule

$p_{1}(d)=p_{i}(d \mid i) \times\left(1-p_{1}(d)\right), d \neq \bar{d}$

$p_{i}(d \mid i)$ : Conditional probability that $b_{i}$ has category $d$ as matching, given that $b_{i}$ is matchable $\left(1-p_{i}(\bar{d})\right)$ : prior probability that $b_{i}$ is matchable

Step 12: Estimate $p_{i}(d \mid i)$ as below

$p_{i}(d \mid i)=\frac{w_{i}(d)}{\sum_{d^{n}=\frac{1}{d} w_{d}} w_{i}\left(d^{3}\right)}$

The new probability $p_{i}^{k+1}(d)$ should tend to increase when descriptors with highly probable category consistent with $d$ are found nearby the key point region. Categories are considered consistent if they represent nearly the same disparity i.e.

$\left|d\left(d_{i}\right)-d\left(d_{m}\right)\right|<\mathrm{T}:$

Where $\mathrm{T}$ is threshold

The threshold has to be decided through trial and error method.

$q_{j}^{k}(d)=\sum_{m=1}^{L} m=j p\left(d_{m}\right)$

$\left|d(d)-d\left(d_{m}\right)\right|<T$

$q_{i j}{ }^{k}(d)$ : Estimated likelihood considering the neighborhood of $d_{j}$, L: Number of neighbors in the category set.

Step 13: Update probability as

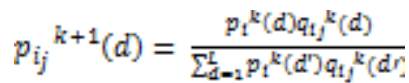

$p_{i}^{k+1}(d)=\sum_{j=1}^{N} \alpha_{j} p_{i j}{ }^{k+1}(d)$

\section{Results and discussion}

In order to validate the image matching capabilities of the proposed approach, 4 subject images having 10 different facial configurations and expressions were tested. 

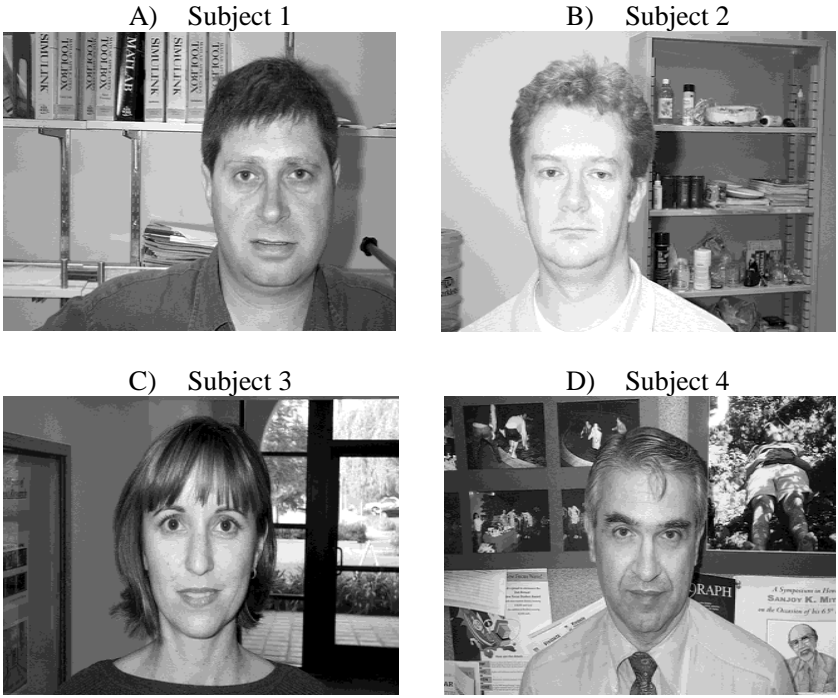

Fig. 4: Images of the Subjects from the Yale Face Data Set Considered for Testing in this Work.

The face recognition algorithm implemented in this work is borrowed from code provided by Lowes [7].A classification approach based on K-d tree algorithm has been implemented. The training setis comprised of 100 images from 10 different subjects. The features of the training images are extracted and stored in a database for the purpose of recognition. A group number is assigned to training images so that an image from same person has the same group number. To further match the image from the test set to the training images, the feature are extracted and each feature of the test image is compared individually with those in the training database. The group number indicates the user on which person has the closest match with the test image. The table 1 to table 4 lists the results of key points identified by SIFT and the proposed image.

Table 1: Key Points Identified By SIFT and the Proposed Approach for Subject 1 for Different Facial Configurations and Expressions

\begin{tabular}{|c|c|c|}
\hline $\begin{array}{l}\text { Subject - } \\
\text { Pose }\end{array}$ & $\begin{array}{l}\text { Number of key points } \\
\text { identified by SIFT }\end{array}$ & $\begin{array}{l}\text { Number of Key points iden- } \\
\text { tified by the proposed ap- } \\
\text { proach }\end{array}$ \\
\hline $\begin{array}{l}\text { Subject } 1- \\
\text { Pose } 1\end{array}$ & 119 & 441 \\
\hline $\begin{array}{l}\text { Subject } 1- \\
\text { Pose } 2\end{array}$ & 204 & 605 \\
\hline $\begin{array}{l}\text { Subject } 1- \\
\text { Pose } 3\end{array}$ & 801 & 379 \\
\hline $\begin{array}{l}\text { Subject } 1 \text { - } \\
\text { Pose } 4\end{array}$ & 102 & 320 \\
\hline $\begin{array}{l}\text { Subject } 1- \\
\text { Pose } 5\end{array}$ & 135 & 358 \\
\hline $\begin{array}{l}\text { Subject } 1- \\
\text { Pose } 6\end{array}$ & 244 & 461 \\
\hline $\begin{array}{l}\text { Subject } 1- \\
\text { Pose } 7\end{array}$ & 129 & 344 \\
\hline $\begin{array}{l}\text { Subject } 1- \\
\text { Pose } 8\end{array}$ & 127 & 217 \\
\hline $\begin{array}{l}\text { Subject } 1- \\
\text { Pose } 9\end{array}$ & 126 & 194 \\
\hline $\begin{array}{l}\text { Subject } 1- \\
\text { Pose } 10\end{array}$ & 122 & 227 \\
\hline
\end{tabular}

Table 2: Key Points Identified By SIFT and the Proposed Approach for Subject 2 for Different Facial Configurations and Expressions

\begin{tabular}{|c|c|c|}
\hline Subject -Pose & $\begin{array}{l}\text { Number of } \\
\text { Key points } \\
\text { identified by } \\
\text { SIFT }\end{array}$ & $\begin{array}{l}\text { Number of } \\
\text { Key points identified by the pro- } \\
\text { posed approach }\end{array}$ \\
\hline $\begin{array}{l}\text { Subject 2- Pose } \\
1\end{array}$ & 118 & 384 \\
\hline${ }_{2}^{\text {Subject 2- Pose }}$ & 148 & 314 \\
\hline $\begin{array}{l}\text { Subject 2- Pose } \\
3\end{array}$ & 199 & 456 \\
\hline $\begin{array}{l}\text { Subject 2- Pose } \\
4\end{array}$ & 108 & 289 \\
\hline $\begin{array}{l}\text { Subject 2- Pose } \\
5\end{array}$ & 114 & 216 \\
\hline $\begin{array}{l}\text { Subject } 2 \text { - Pose } \\
6\end{array}$ & 120 & 251 \\
\hline $\begin{array}{l}\text { Subject 2- Pose } \\
7\end{array}$ & 131 & 356 \\
\hline $\begin{array}{l}\text { Subject 2- Pose } \\
8\end{array}$ & 127 & 419 \\
\hline $\begin{array}{l}\text { Subject 2- Pose } \\
9\end{array}$ & 105 & 359 \\
\hline $\begin{array}{l}\text { Subject 2- Pose } \\
10\end{array}$ & 122 & 463 \\
\hline
\end{tabular}

Table 3: Key Points Identified By SIFT and the Proposed Approach for Subject 3 for Different Facial Configurations and Expressions

\begin{tabular}{lll}
$\begin{array}{l}\text { Subject - } \\
\text { Pose }\end{array}$ & $\begin{array}{l}\text { Number of key points } \\
\text { identified by SIFT }\end{array}$ & $\begin{array}{l}\text { Number of } \\
\text { Key points identified by the } \\
\text { proposed approach }\end{array}$ \\
\hline $\begin{array}{l}\text { Subject 3- } \\
\text { Pose 1 }\end{array}$ & 113 & 222 \\
$\begin{array}{l}\text { Subject 3- } \\
\text { Pose 2 }\end{array}$ & 117 & 287 \\
$\begin{array}{l}\text { Subject 3- } \\
\text { Pose 3 }\end{array}$ & 126 & 367 \\
$\begin{array}{l}\text { Subject 3- } \\
\text { Pose 4 }\end{array}$ & 139 & 365 \\
$\begin{array}{l}\text { Subject 3- } \\
\text { Pose 5 } \\
\text { Subject 3- }\end{array}$ & 113 & 369 \\
$\begin{array}{l}\text { Pose 6 } \\
\text { Subject 3- } \\
\text { Pose 7 } \\
\text { Subject 3- }\end{array}$ & 130 & 368 \\
$\begin{array}{l}\text { Pose 8 } \\
\text { Subject 3- } \\
\text { Pose 9 } \\
\text { Subject 3- }\end{array}$ & 130 & 300 \\
Pose 10 & 149 & 198 \\
\hline
\end{tabular}

Table 4: Key Points Identified By SIFT and the Proposed Approach for Subject 4 for Different Facial Configurations and Expressions

\begin{tabular}{lll} 
Subject -Pose & $\begin{array}{l}\text { Number of } \\
\text { Key points } \\
\text { I identified } \\
\text { by SIFT }\end{array}$ & $\begin{array}{l}\text { Number of } \\
\text { Key points identified by } \\
\text { the proposed approach }\end{array}$ \\
\hline Subject 4- Pose 1 & 163 & 385 \\
Subject 4- Pose 2 & 171 & 441 \\
Subject 4- Pose 3 & 112 & 548 \\
Subject 4- Pose 4 & 176 & 272 \\
Subject 4- Pose 5 & 167 & 310 \\
Subject 4- Pose 6 & 187 & 218 \\
Subject 4- Pose 7 & 176 & 475 \\
Subject 4- Pose 8 & 142 & 450 \\
Subject 4- Pose 9 & 178 & 353 \\
Subject 4- Pose 10 & 182 & 247 \\
\hline
\end{tabular}

Subsequently the images are matched and the results of the identifications are presented here 
Table 5: Performance Comparison of the Proposed Detector and the Detector Based on SIFT

\begin{tabular}{lllll}
\hline & \multicolumn{2}{l}{ SIFT based detection } & \multicolumn{2}{l}{$\begin{array}{l}\text { Proposed method based detec- } \\
\text { tion }\end{array}$} \\
$\begin{array}{l}\text { Sub- } \\
\text { ject }\end{array}$ & $\begin{array}{l}\text { Number of } \\
\text { True identi- } \\
\text { fications }\end{array}$ & $\begin{array}{l}\text { Number of } \\
\text { False identi- } \\
\text { fications }\end{array}$ & $\begin{array}{l}\text { Number of } \\
\text { True identi- } \\
\text { fications }\end{array}$ & $\begin{array}{l}\text { Number of } \\
\text { False identi- } \\
\text { fications }\end{array}$ \\
$\begin{array}{l}\text { Sub- } \\
\text { ject } 1\end{array}$ & 10 & 0 & 10 & 0 \\
$\begin{array}{l}\text { Sub- } \\
\text { ject } 2\end{array}$ & 10 & 0 & 10 & 0 \\
$\begin{array}{l}\text { Sub- } \\
\text { ject } 3\end{array}$ & 10 & 0 & 10 & 0 \\
$\begin{array}{l}\text { Sub- } \\
\text { ject } 4\end{array}$ & 10 & 0 & 10 & 0 \\
\hline
\end{tabular}
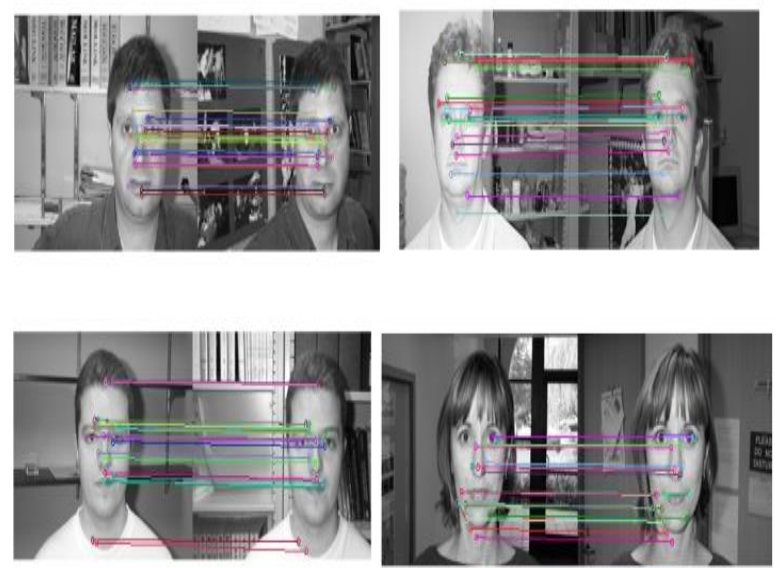

Fig. 5: Results of Image Matching by the Proposed Approach for Different Subjects.

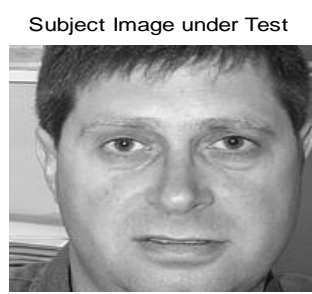

This image matches and corresponds to Subject 1

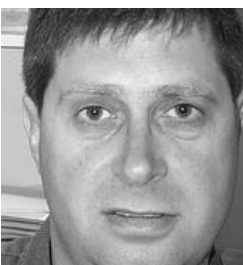

Fig. 6: Sample Display of Result for Image Recognition Using the Proposed Approach.

It can be observed from the results that the proposed approach performs well in comparison to SIFT based detection of facial images. The performance is visible across the spectrum. This is especially visible in regard to the number of key points being identified. It can be inferred from the table 1 to table 4 the number of key points identified by the proposed approach is significantly higher than the SIFT descriptor. Similarly in order to validate the performance a test data set was formed with images from the training data and also images which are not part of the training data set. The results of this classification are illustrated with the help of the following table. This data set is comprised of a total of 10 images with 4 images which are part of training data and 6 images which are not part of training data. For those images which are part of training images, a correct match is counted when the image is matched correctly. For those images which are not part of the data set, a correct match is when the algorithm identifies that it's not part of the heterogeneous dataset.
Table 6: Performance Comparison of the Proposed Detector and the Detector Based on SIFT for Images in Which 4 Images are Part of Training Data and 6 are Not Part of Training Data

\begin{tabular}{|c|c|c|c|}
\hline Method & $\begin{array}{l}\text { Number of } \\
\text { Correct Match- } \\
\text { es }\end{array}$ & $\begin{array}{l}\text { Number of } \\
\text { False Matches }\end{array}$ & $\begin{array}{l}\text { Accuracy } \% \text { of } \\
\text { recognition }\end{array}$ \\
\hline $\begin{array}{l}\text { SIFT Based } \\
\text { Detection }\end{array}$ & 4 & 6 & 40 \\
\hline $\begin{array}{l}\text { Proposed } \\
\text { Approach }\end{array}$ & 9 & 1 & 90 \\
\hline
\end{tabular}

Table 5 presents the matching results for SIFT and the proposed approach for face recognition of the subjects. This table 5 incorporates the results for the images which are in the training set, for this case the face recognition accuracy by both the approaches are very high. In fact both the approaches have exhibited 100 percent accuracy in recognizing the faces. In order to establish the robustness of the approach in identifying the images that are not part of training data the experiments were carried out and results tabulated in table 6. It can be observed from the results that for this case there is a significant fall in the accuracy of face recognition for this test set for SIFT. On the other hand the proposed approach delivered an accuracy of $90 \%$. This can be attributed to the high number of descriptor points identified by the proposed approach. This automatically enhances the chances of accurate matching.

\section{Conclusion}

A new frame work for view and illumination invariant image matching has been designed and effectively presented for application in face matching. The performance of the proposed algorithm was evaluated with images from Yale face data set. It can be clearly inferred from the results that the proposed approach has outperformed the SIFT in terms of identifying the number of image matches and deliver an enhanced accuracy for detection in a heterogeneous data set. Even though it was not explicitly documented, it was clearly observed during the execution the proposed approach had a much smaller execution time. This can be attributed to the reduced computational complexity making it suitable for real time applications like face recognition.

\section{References}

[1] R. Jafri and H.R.Arabnia(2009)A Survey of Face RecognitionTechniques, Journal of Information Processing Systems, Vol.5, no.2, pp. 41-68. https://doi.org/10.3745/JIPS.2009.5.2.041.

[2] W.Zhao, R. Chellappa, P.Philips, and A. Rosenfeld (2003) Face recognition: A literature survey,"ACMComputing Survey, Vol.35, no.4, pp. 399-458. https://doi.org/10.1145/954339.954342.

[3] Y.Taigman,M.Yang,M.Ranzato, L.Wolf (2014) Closing The Gap to Human Level Performance in Face Verification,Int. Conference on Computer Vision and Pattern Recognition (CVPR),,pp.1701-1708. https://doi.org/10.1109/CVPR.2014.220.

[4] M. Turk, A. Pentland (1991) Eigen faces for Recognition, Cognitive Neuroscience, Vol. 3, no. 1, pp. 71-86. https://doi.org/10.1162/jocn.1991.3.1.71.

[5] C. Xiang, X. Fan, and T. Lee (2006) Face Recognition UsingRecursive FisherLinear Discriminant, IEEE Transactions on Image Processing, Vol. 15, no. 8, pp. 2097-2105. https://doi.org/10.1109/TIP.2006.875225.

[6] C. Liu(2006)Capitalize On Dimensionality Increasing Techniques for Improving Face Recognition Grand Challenge Performance, IEEE Transactions on Pattern Analysis and Machine Intelligence, Vol. 28, no. 5, pp. 725-737. https://doi.org/10.1109/TPAMI.2006.90.

[7] D.G. Lowe (1999) Object Recognition from Local Scale-Invariant Features, Proceedings of the International Conference on Computer Vision, Vol. 2, Sept. 20-25, pp. 1150-1157. https://doi.org/10.1109/ICCV.1999.790410.

[8] S. Se, D. Lowe, and J. Little(2001)Vision-Based Mobile Robot Localization and Mapping Using Scale-Invariant Features, Proceedings of the IEEE Conference on Robotics and Automation, Vol. 2, pp. 2051-2058. https://doi.org/10.1109/ROBOT.2001.932909. 
[9] T. Pham, N. Waillot, J. Lim, J. Chevallet (2007)Latent Semantic Fusion Model For Image Retrieval and Annotation, Proceedings of the ACM Conference on Information and Knowledge Management, pp. 439-444.

[10] M. Bicego, A. Lagorio, E. Grosso, M. Tistarelli (2006) On The Use Of Sift Features For Face Authentication, Proceedings of the Conference on Computer Vision and Pattern Recognition Workshop, pp. 35-41. https://doi.org/10.1109/CVPRW.2006.149.

[11] C. Geng and X. Jiang (2009) Face Recognition using SIFT Features, Proceedings of the International Conference onImage Processing, pp. 3313-3316. https://doi.org/10.1109/ICIP.2009.5413956.

[12] D. Kisku, A. Rattani, E. Grosso and M. Tistarelli (2010)Face Identification by SIFT-based Complete Graph Topology, CoRRabs/1002.0411.

[13] Jnez Križaj,,Vitomir Štruc,,andNikola Pavešić(2010)Adaptation of SIFT Features for Robust Face Recognition, ICIAR,Springer, Heidelberg, pp. 394-404.

[14] J. Luo, Y. Ma, E. Takikawa, S. Lao, M. Kawade, B. L. Lu (2007) Person-specific SIFT Features for Face Recognion, IEEE International Conference on Acoustics, Speech and Signal Processing, Vol. 2, pp. 593-596.

[15] N. Kumar, A. Berg, P. Belhumeur, S. Nayar (2009) Attribute and Simile Classifiers for Face Verification, Proceedings of the International Conference on Computer Vision, pp. 365-372. https://doi.org/10.1109/ICCV.2009.5459250.

[16] D.G. Lowe (2004) Distinctive Image Features from Scale- Invariant KeyPoints, International Journal of Computer Vision, Vol. 60, no. 2, pp.91-110. https://doi.org/10.1023/B:VISI.0000029664.99615.94.

[17] H.Bay,A.Ess,T.Tuytelaars,L.Van Gool (2008) Speeded-UpRobus Features (SURF),ComputerVision and Image Understanding, Vol.110, no. 3,pp.346-359. https://doi.org/10.1016/j.cviu.2007.09.014.

[18] M.Calonder, V.Lepetit, C.Strecha, P.Fua (2010) BRIEF: Binary Robust Independent Elementary Features, Daniilidis. K., Maragos. P,Paragios. N(eds.) ECCV, Part IV. LNCS, vol. 6314, Springer, Heidelberg, pp. 778-792

[19] http://vision.ucsd.edu/datasets/yale_face_dataset_original/yalef aces.zip

[20] Paul Viola and Michael Jones(2010) Rapid Object Detection using a Boosted Cascade of Simple Features, Artificial Intelligence Journal, Vol. 78, pp. 507-545.

[21] http://docs.opencv.org/2.4/doc/user guide/ug traincascade.html.

[22] Dr. Seetaiah Kilaru, Hari Kishore K, Sravani T, Anvesh Chowdary L, Balaji T "Review and Analysis of Promising Technologies with Respect to fifth Generation Networks", 2014 First International Conference on Networks and Soft Computing, ISSN:978-1-4799-3486-7/14,pp.270-273,August2014.

[23] T. Padmapriya and V. Saminadan, "Improving Throughput for Downlink Multi user MIMO-LTE Advanced Networks using SINR approximation and Hierarchical CSI feedback", International Journal of Mobile Design Network and Innovation- Inderscience Publisher, ISSN : 1744-2850 vol. 6, no.1, pp. 14-23, May 2015.

[24] S.V.Manikanthan and K.srividhya "An Android based secure access control using ARM and cloud computing", Published in: Electronics and Communication Systems (ICECS), 2015 2nd International Conference on 26-27Feb. 2015, Publisher: IEEE https://doi.org/10.1109/ECS.2015.7124833. 